\title{
A nine-year-old patient affected by chromosomal aberration with the suspicion of juvenile idiopathic arthritis
}

\author{
Zbigniew Żuber, Magdalena Nawrotek, Małgorzata Sobczyk, Elżbieta Mężyk, \\ Dorota Turowska-Heydel
}

Department of Older Children with Subunits of Neurology, Rheumatology and Rehabilitation, St. Louis Regional Specialised Children's Hospital, Krakow, Poland

\begin{abstract}
We present a case study of a 9.5-year-old girl affected by chromosome 12 aberration with the suspicion of juvenile idiopathic arthritis (JIA). All the tests performed at the hospital and the presence of a genetic disorder ledus to search for a diagnosisother than JIA. We initially diagnosed spondyloepiphyseal dysplasia tarda (SEDT), which is related to chromosome 12. Certain signs and symptoms of this disease were presented by our patient at the time of admission. After analysing all the tests and the general conditionof the patient, we were unable to confirm this diagnosis. However, it is possible that the symptoms may occur during subsequentyears and may allow confirmation of SEDT in the future.
\end{abstract}

Key words: arthritis, juvenile idiopathic arthritis, diagnostic, chromosomal aberration.

\section{Case presentation}

A 9.5-year-old girl with gait disorder and presenting swelling of the knee and wrist was admitted to the Neurology and Rheumatology Department with the suspicion of juvenile idiopathic arthritis (JIA). This patient with chromosomal aberration 46,XX inversion of the loci (12)(q15;q13.1) was affected by ventricular septal defect (VSD), epilepsy, low muscle tension, skeletal abnormalities, cleft palate, persistent urogenital sinus, strabismus, and dysmorphia. This child was delivered in the $35^{\text {th }}$ week of gestation, G1P1 (gravidity 1, partus 1), with birth weight $2200 \mathrm{~g}$, height $51 \mathrm{~cm}$, head circumference $31 \mathrm{~cm}$, and the Apgar score at first minute was 6. Neither of the parents or other family members had medical conditions or genetic risk. Due to epilepsy the patient was treated with valproic acid and levetiracetam, with good control of the disease. Two months before hospital admission the patient was hospitalised at the Emergency Department due to swelling of the left wrist and small joints of the hands. There was no trauma or insect bite during the few days before. The last infection that needed anti- biotic treatment occurred two months earlier, so the patient was discharged with the recommendation to use non-steroidal anti-inflammatory drugs (NSAIDs) topically. The symptoms subsided without any other medical intervention. There was no fever, eczema, joint pain, or arthritis untilthe time of admission to the Rheumatology Department when the knee swelling appeared.

The patient was in good general condition, presented mild mental retardation, poor muscle power and tone, varus feet and valgus knees, limited mobility of the right wrist, left knee with a largeamount of fluid, limited mobility and painful when flexing, abnormal patella position in both extremities (lateralisation of the patella), and barrel-shaped chest. Moreover, the patient had dysmorphic features with right eye ptosis, epicanthic fold, abnormal patterns on the fingertips, short fingers and toes with toes syndactyly, delayed eruption of adult teeth, shorter roots on teeth, and malformed and missing teeth. The medical history of this patient included surgical reconstruction of the craniofacial region due to a cleft palate, and asystolic murmur (grade $3 / 6$ in Levine' scale) was found. The rest of the patient's 
respiratory, cardiovascular, and gastrointestinal systems showed no deviations. Vision and hearing was normal, weigh was $34.5 \mathrm{~kg}$ (between $50^{\text {th }}$ and $75^{\text {th }}$ centile), and height was just short $127 \mathrm{~cm}$ on the $3^{\text {rd }}$ centile.

The JIA was suspected based on the observed symptoms of the musculoskeletal system. To confirm the diagnosis and exclude other causes of arthritis we performer laboratory tests, X-ray imaging, and ultrasound examination of the swollen joints. Haematological and serological investigation showed no evidence of rheumatic disease. The level of interferon $\gamma$ was reduced $(11.58 \mathrm{pg} / \mathrm{ml}$; norm 1000-1500 pg/ml), and the level of tumour necrosis factor $\alpha$ (TNF- $\alpha$ ) was low (158.06 pg/ml; norm 750-1500 pg/ml). Other immunological tests, i.e.: anti-citrullinated protein antibodies (ACPA), rheumatoid factor (RF), and antinuclear antibodies (ANA) were negative. Also, we did not confirm the presenceof HLA-B27.

Radiographs of the spine, feet, and hands showed mild osteopaenia, there were no widened ends of tubular bones, and no signs of bone destruction such as erosions. The joint spaces and the intervertebral disc spaces of the cervical, thoracolumbar spine were normal. Also, there was no evidence of platyspondyly. However, radiographic studies of the spine revealed thoracolumbar kyphosis and scoliosis. Lower-limb radiographs showed marked lateral condylar hypoplasia with lateralisation (subluxation) of both patellae, as shown in Figure 1 and Figure 2.

The hip X-ray showed moderate dysplasia and flattened femoral heads with normal joint space of both hips. Knee and hip ultrasound examination revealed a lack of synovial hyperplasia or active synovial hyperaemia (power Doppler was evaluated). There were no abnormalities in ECG, lung X-ray, and ophthalmological

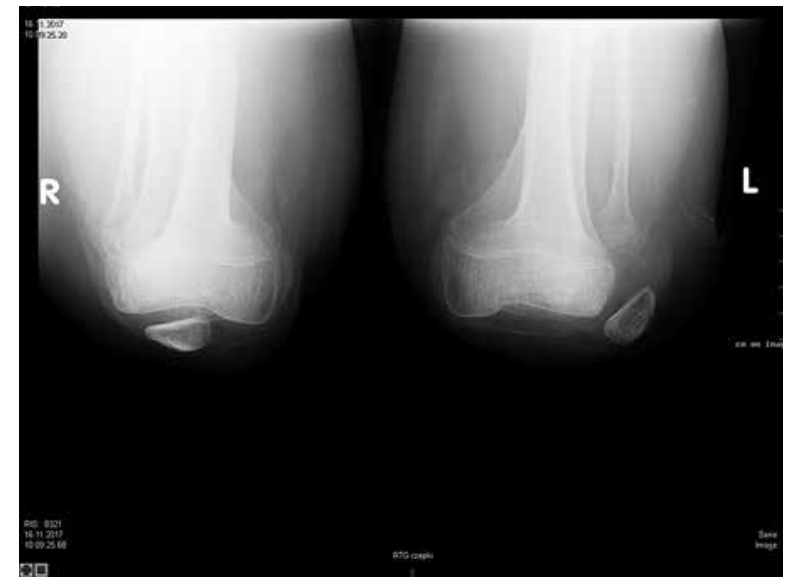

Fig. 1. Lateral condylar hypoplasia with lateralisation of patellae (with a predominance on the left side) - flexion. examination. Echocardiography revealed sub-aortic VSD with no haemodynamic significance. We performed knee puncture with steroid intra-articular injection. There were no signs of any inflammation in the collected fluid.

After performing the clinical examination and analysing the laboratory tests, the suspicion of the diagnosis of the JIA was rejected [1, 2]. For the diagnosis of JIA, the patient's age and joint symptoms of many joints, including minor joints, lasting more than six weeks were typical, but the absence of inflammatory factors in blood and knee-fluid and the presence of skeletal abnormalities connected with chromosome abnormality led us to search for a diagnosis other than JIA. In this case, joint symptoms such as arthralgia or arthritis may have appeared as an effect of skeletal abnormalities, or they might be the part of the genetic pattern.

\section{Discussion}

Because the patients has been diagnosed with chromosome 12 inversion of the loci (q15; q13.1), we suspected that some of the symptoms could be related to this aberration. The parents' DNA was also examined in order to find any hereditary abnormalities. Since neither of them transpired to have genetic risks, the aberration of the child's genome was described as a de novo condition. Table I shows genes and conditions associated with loci of chromosome 12, the aberrations of which are described in this case study. The patient presented few symptoms, which may be explained by chromosomal aberration, e.g. mental retardation or reduced level of interferon $\gamma($ IFN- $\gamma)$. But the most interesting was finding locus 12 q13.1 to be related to spondyloepiphyseal dysplasia tarda (SEDT). It attrated our attention as a possible explanation of the patient's condition.

Spondyloepiphyseal dysplasia is a group of genetic disorders that mostly affects the skeleton and may be transmitted as an X-linked recessive, autosomal recessive, or autosomal dominant trait. The one associated with chromosome 12 is usually transmitted as AD and

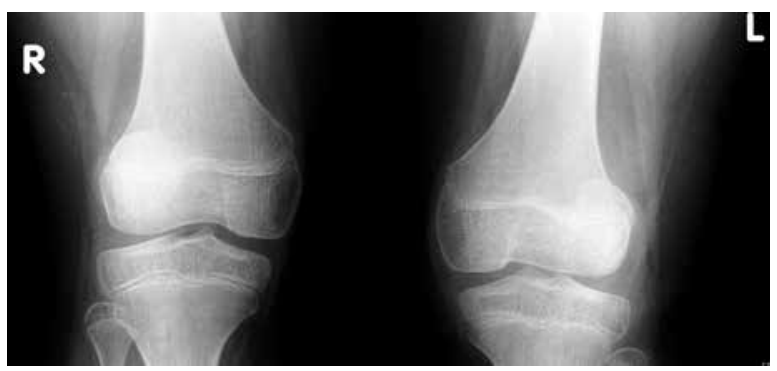

Fig. 2. Lateral condylar hypoplasia with lateralisation of patellae - AP/Long Leg Views. 
Table I. Spondyloepiphyseal dysplasia tarda

\begin{tabular}{|c|c|}
\hline $12 q 13.1$ & $12 q 15$ \\
\hline Susceptibility to basal cell carcinoma & Inflammatory bowel disease \\
\hline Susceptibility to chronic mountain sickness & $\begin{array}{l}\text { IFN- } \gamma \text { - rapid progression to AIDS, response to therapy of HCV, aplastic anaemia, } \\
\text { angiomyolipomas, protection against tuberculosis }\end{array}$ \\
\hline Deafness AD & IL-22, IL-26 \\
\hline Enuresis nocturnal & RAS-related protein RAP1B \\
\hline HPV type 18 integration site-2 & Nucleoporin - nephrotic syndrome type 11 \\
\hline Mental retardation $A R$ & Carboxypeptidase $M$ \\
\hline Spondyloepiphyseal dysplasia tarda AD & \\
\hline
\end{tabular}

$A D$ - autosomal dominant; $A R$ - autosomal recessive

may be linked with the aberrations of the COL2A1 gene. Its symptoms include many skeletal abnormalities that may be not present at birth. The progression of the disease is usually correlated with increased bone growth during adolescence and affects body structure, which results in short trunk, reduced upper-to lower-body segment ratio, and a barrel shaped chest. The defects of the spine include platyspondyly, thoracic kyphosis or scoliosis, lumbar hyperlordosis, hypoplasia of the odontoid process, pectus carinatum, or irregular vertebral endplates. The rest of the symptoms affect the extremities: abnormality of epiphysis morphology, coxa vara, hypoplastic iliac wing, avascular necrosis of the capital femoral epiphysis, cervical subluxation, congenital hip dislocation, and knee and hip arthralgia. All the described defects may lead to arthritis with joint swelling and pain or gait disorders.

Many rare genetic disorders affecting the skeleton may clinically present very similarly to JIA, and therefore it is important to remember about them [3-5]. The ILAR criteria allow diagnosis of JIA only after excluding other causes [1, 2]. The genetic skeleton disorders should be taken under consideration, especially if the patient presents certain symptoms. Robinson et al. referred two siblings suffering from spondyloepiphyseal dysplasia with progressive arthropathy (SEDT-PA), a form of SED. The symptoms included normal mental development, progressive flexion contractures, spine abnormalities, and mild arthritis.

The inflammation parameters were normal. Another patient with SEDT-PA described by Arslaanoglu [6] also presented typical SED symptoms including platyspondyly, arthritis with no laboratory evidence of inflammation, and normal intelligence. Lankisch et al. [7] referred a case report about a family with conditions related to few COL2A1 mutations. Each of them resulted in different symptoms such as height and face disorders, hearing loss, ocular features, cleft palate, arthropathy, and skeletal abnormalities. Every collagen genetic syndrome results in another phenotype, including SED. Collag- enopathies may have onset in the intrauterine, newborn, childhood, or adult period [8].

Each member of this group of disorders is connected with a particular disease and leads to various disability levels, including achondrogenesis, which is lethal. Unlike in our case, the mental development is generally not disturbed. However, Kohn et al. [9] described a case of three sisters suffering from SEDT, who presented mild to moderate mental retardation. The described patients presented only part of SEDT or collagenopathy symptoms. Radiograms did not reveal platyspondyly, which is supposed to be the most characteristic feature of SED $[3,5,10,11]$. However, it is possible that in this patient other symptoms of SEDT may develop in future. Sahin [11] described a case of a boy with SEDT with progression of disease symptoms for several years. If a patient does not fulfill the criteria SEDT the possibility of other, not yet described mutations should be considered [12]. Radiograms taken at age eight and nine years in our patient revealed significant progression and expansion of platyspondyly during these four years.

\section{Conclusions}

The described patient has no simple point mutation but a complicated chromosome aberration, which may result in many different symptoms. The diagnosis of SEDT is one of the possible explanations for the patient's condition. Further genetic diagnostics (for example, to find collagen abnormalities) may be taken under consideration. Rehabilitation and regular paediatric control will be necessary at further stages of the child's growth.

The authors declare no conflict of interest.

\section{References}

1. Petty R, Southwood T, Baum J, et al. Revision of the proposed classification criteria for juvenile idiopathic arthritis: Durban 1997. J Rheumatol 1998; 25: 1991-1994. 
2. Weiss J, Ilowite N. Juvenile idiopathic arthritis. Pediatr Clin North Am 2005; 52: 413-442.

3. Robinson D, Tieder M, Haiperin N, et al. Spondyloepiphyseal Dysplasia Associated with Progressive Arthropathy. Arch Orthop Trauma Surg 1989; 108: 397-399.

4. Korkmaz C, Kasifogl T, Akay OM. Spondyloepiphyseal dysplasia tarda simulating juvenile chronic arthritis. Clin Exp Rheumatol 2003; $21: 677$

5. Chung SW, Kang EH, Lee YJ. Three Cases of Spondyloepiphyseal Dysplasia Tarda in One Korean Family. Yonsei Med J 2016; 57: 1290-1293.

6. Arslanoglu S, H. Murat H, Ferah G. Spondyloepiphyseal dysplasia tarda with progressive arthropathy: An important form of osteodysplasia in the differentia diagnosis of juvenile rheumatoid arthritis. Pediatrics International 2000; 42: 561-563.

7. Lankisch P, Hönscheid A, Schaperc J, et al. COL2A1 mutation as a cause of premature osteoarthritis in a 13-year-old child. Joint Bone Spine 2014; 81: 83-85.

8. Kannu P, Bateman J, Savarirayan R. Clinical phenotypes associated with type II collagen mutations. J Paediatr Child Health 2012; 48: E38-E43.

9. Kohn G, Elrayyes ER, Makadmah I. Spondyloepiphyseal dys plasia tarda: a new autosomal recessive, variant with menta retardation. J Med Genet 1987; 24: 366-377.

10. Bal S, Kocyigit H, Turan Y, et al. Spondyloepiphyseal dysplasia tarda: four cases from two families. Rheumatol Int 2009; 29 699-702.

11. Sahin AO, Bolukbasi N, Beyazowa M. Spondyloepiphyseal dysplasia tarda in a child with severe and an adult with mild clinical features. Clin Exp Rheumatol 2001; 19: 481.

12. Jurgens J, Sobreira N, Modaff P. Novel COL2A1 variant (c.619G >A, p.Gly207Arg) manifesting as a phenotype similar to progressive pseudorheumatoid dysplasia and spondyloepiphyseal dysplasia, Stanescu type. Hum Mutat 2015; 36 1004-1008. 